\title{
Menschenrechtsmonitoring im Kinder- und Jugendbereich
}

\author{
Ernst Berger (D) C Caroline Paar
}

Eingegangen: 6. April 2017 / Angenommen: 29. Juli 2017 / Online publiziert: 30. August 2017

(C) Der/die Autor(en) 2017. Dieser Artikel ist eine Open-Access-Publikation.

Zusammenfassung Mit der Etablierung des Nationalen Präventionsmechanismus (NPM) durch das OPCAT-Gesetz wurden in Österreich im Jahre 2012 sechs Menschenrechtskommissionen bei der Volksanwaltschaft eingerichtet, die $u$. a. kinder- und jugendpsychiatrische Krankenhausabteilungen sowie Wohneinrichtungen der Kinder- und Jugendhilfe besuchen. Aus dem Bereich der Kinder- und Jugendpsychiatrie liegen 20 Besuchsprotokolle aus der Zeitperiode 2012-2016 vor. Im Kinder- und Jugendhilfebereich wurden 40 Besuchsprotokolle (von insgesamt 176 der Jahre 2015-2016) ausgewählt. Die vorliegende Arbeit gibt einen Überblick über die Rechtsgrundlagen des Monitoringprozesses, über die Monitoringthemen sowie über die Inhalte der Besuchsprotokolle.

Schlüsselwörter Menscherechtsmonitoring - OPCATGesetz · Kinderrechtekonvention · Freiheitsbeschränkungen · Recht auf Partizipation · Kinder- und Jugendhilfe $\cdot$ Gewaltprävention

\section{Preventive human rights monitoring in child and} adolescent psychiatry and welfare institutions

Summary Since 1 July 2012 the Austrian Ombudsman Board (AOB) together with its six regional expert commissions form the so called "National Prevention Mechanism" implementing the UN Optional Protocol

Der interdisziplinäre Charakter des Themas macht es unumgänglich, von üblichen Formatvorgaben abzuweichen. Relevante inhaltliche Informationen aus dem juridischen Bereich werden in Fußnoten dargestellt.

\footnotetext{
E. Berger $(\bowtie)$

Loudonstraße 40A, 1140 Wien, Österreich

ernst.berger@meduniwien.ac.at

C. Paar

Antonsgasse 19/9/4, 2500 Baden, Österreich
}

to the Convention against Torture and other Cruel, Inhuman or Degrading Treatment or Punishment (OP$\mathrm{CAT}$ ). The commissions are mandated to conduct regular, unrestricted and unannounced visits to all types of places in which there is or can be a deprivation or restriction of personal liberty, such as prisons, police stations, but also psychiatric hospitals or residential homes/groups for children and juveniles supervised by youth welfare services. 20 monitoring visits have been held in departments for Child- and Adolescent Psychiatry between 2012 and 2016. In the field of residential groups for young people 40 visiting protocols (out of a total of 176 between 2015 and 2016) have been chosen for this analysis. The following article overviews the legal sources and key issues of the monitoring process in this field as well as the commissions' findings and recommendations.

Keywords Preventive human rights monitoring - Act of the implementation of the OPCAT - Children's Rights Convention - Restriction of liberty - Right to participation - Welfare for children and adolescents . Violence prevention

\section{Einleitung und Ausgangslage}

Das OPCAT-Durchführungsgesetz beauftragt die Volksanwaltschaft und ihre Kommissionen, „den Ort einer Freiheitsentziehung zu besuchen und $\mathrm{zu}$ überprüfen“. Psychiatrische Abteilungen zählen somit zu den Kernbereichen dieses Prüfauftrages, da Freiheitsentziehung - dem Unterbringungsgesetz entsprechend einen Teil des psychiatrischen Alltags darstellt. Dies trifft vollinhaltlich auch für kinder- und jugendpsychiatrische Abteilungen und im weiteren Sinne auch auf sozialpädagogische Einrichtungen zu, da sie Träger des Vollzugs jugendhilferechtlicher Maßnahmen sind. 
Für alle derartigen Einrichtungen wurde durch das Inkrafttreten des OPCAT-Gesetzes im Jahre 2012 eine völlig neue Situation geschaffen: Kommissionen der Volksanwaltschaft kommen zu unangekündigten Monitoringbesuchen und überprüfen die Alltagspraxis. Diese Besuche beruhen auf verfassungsrechtlichen und einfachgesetzlichen Bestimmungen vor dem Hintergrund eines Zusatzprotokolls zur UN-Antifolter-Konvention ${ }^{1}$, über dessen Einhaltung ein UNSubkomitee wacht. ${ }^{2}$ Da weder in Wohneinrichtungen der Jugendhilfe noch in kinderpsychiatrischen Krankenhausabteilungen ein direkter Bezug zum Titel der UN-Konvention („Folter und andere grausame, unmenschliche oder erniedrigende Behandlung“) ersichtlich ist, erscheint es wenig erstaunlich, dass die Monitoringbesuche vorerst skeptisch betrachtet wurden. Der Begriff des „Nationalen Präventionsmechanismus“ (NPM), der sowohl auf der UN-Ebene als auch in den erläuternden Bemerkungen zum österreichischen OPCAT-Gesetz verwendet wird, betont den präventiven Charakter des Monitorings, der mit der Tätigkeit dieser Einrichtungen wesentlich besser kompatibel ist. Ein Blick in die Geschichte [1, 3, 4 , 7] macht jedoch deutlich, dass zu den Begriffen „grausame, unmenschliche und erniedrigende Behandlung “ in der Vergangenheit durchaus ein realer Bezug bestand. Die „Periode der Anstalten“ [5] war geprägt durch Einrichtungen, die dem Typus der „totalen Institution“ [11] entsprachen. Die Entwicklung der letzten Jahrzehnte hat zu grundlegenden Wandlungen geführt. Die Kinder- und Jugendhilfe stützt sich heute auf kleine Wohneinheiten, die in den Alltag des städtischen (und auch ländlichen) Lebens integriert sind; die Kinder- und Jugendpsychiatrie ist (mit großer Verspätung) seit 2007 ein eigenständiges medizinisches Sonderfach mit eigenen (ambulanten und stationären) Versorgungsstrukturen [6]. Dennoch ist die externe Evaluation des Alltagsbetriebs dieser Einrichtungen anhand menschenrechtlicher Standards ein wesentliches Element der Qualitätssicherung. Nach vier Jahren der praktischen Umsetzung soll in diesem Beitrag über die Erfahrungen berichtet werden.

\section{Methode}

Der Erfahrungsbericht stützt sich auf die Auswertung der Besuchsprotokolle ${ }^{3}$ der 6 Kommissionen der Volksanwaltschaft (aus ganz Österreich), die im Anschluss an einen Kommissionsbesuch von jenen Mitgliedern der interdisziplinär zusammengesetzten

\footnotetext{
1 Optional Protocol to the Convention against Torture and other Cruel, Inhuman or Degrading Treatment or Punishment, UN 2006 (OPCAT).

2 Subcomitee on Prevention of Torture (SPT).

3 Die Beobachtungen werden in Protokollformularen festgehalten https://volksanwaltschaft.gv.at/downloads/dvnqf/Besuchs protokoll.pdf (download 28.03.17).
}

Kommission, die am Besuch beteiligt waren, kooperativ erstellt, vom Kommissionsleiter finalisiert und freigegeben und an die Volksanwaltschaft übermittelt werden. Der Protokollinhalt ist ein freier narrativer Fließtext, der - dem Protokollformular folgend - eine systematische Gliederung aufweist. Da eine quantitative Auswertung dieser Protokolle nicht sinnvoll ist, erfolgte die Evaluation der Protokolle auf der Grundlage der qualitativen Inhaltsanalyse nach Mayring [13]. Die Haupt- und Subkategorien wurden induktiv (im Sinne der zusammenfassenden Inhaltsanalyse) aus den Protokolltexten gewonnen. Aus dem Bereich der Kinder- und Jugendpsychiatrie liegen 20 Besuchsprotokolle aus der Zeitperiode 2012-2016 vor, die zur Gänze in die Evaluation einbezogen wurden. Im Kinder- und Jugendhilfebereich liegen aus dem Zeitraum 2015-2016 insgesamt 176 Protokolle vor, aus denen 40 Besuchsprotokolle $^{4}$ so ausgewählt wurden, dass ein typischer Querschnitt der verschiedenen Einrichtungstypen dargestellt wird. Aufgrund der Heterogenität der Einrichtungstypen in diesem Bereich musste auf eine Kategorienbildung verzichtet und eine überblicksmäßige Darstellungsform gewählt werden.

\section{Grundlagen des menschenrechtlichen Monitoring}

Die Menschenrechte, die von den Vereinten Nationen in der „Allgemeinen Erklärung der Menschenrechte“ (AEMR, 1948) sowie vom Europarat in der „Europäischen Menschenrechtskonvention“ (EMRK, 1950) formuliert wurden, gelten für Kinder und Jugendliche gleichermaßen wie für alle anderen Menschen. Die „Kinderrechtekonvention“ (UN-KRK, 1989) - eine kinderspezifische Weiterentwicklung der AEMR - wurde von Österreich 1992 ratifiziert. Sie definiert als „Kind“ grundsätzlich alle Menschen, die das 18. Lebensjahr noch nicht vollendet haben, sofern die Volljährigkeit nach anzuwendendem Recht nicht früher eintritt. Knapp 20 Jahre später trat ein Verfassungsgesetz in Kraft, das wesentliche Teile der UN-KRK übernommen hat - das BVG Kinderrechte [9]. Damit hat Österreich einen verfassungsrechtlichen Schutz der Kinderrechte geschaffen.

\footnotetext{
${ }^{4}$ Insgesamt haben die Kommissionen in den beiden Jahren 176 Einrichtungen der Kinder- und Jugendhilfe besucht. Die hier referierte Auswahl repräsentiert das Spektrum unterschiedlicher Einrichtungstypen: Großeinrichtungen (wie Landesjugendheime) und Kleinstgruppen, Einrichtungen öffentlicher wie privater Träger, unterschiedliche Bundesländer, ländliche und (groß)städtische Regionen, unterschiedliche Altersstufen und unterschiedliche Betreuungsintensität/Konzeption der Wohngruppen (sozialpädagogische, intensivpädagogische, sozialtherapeutische und sozialpsychiatrische). Erfasst wurden ebenso einzelne Einrichtungen an der Schnittstelle zur Behindertenhilfe. Ausgeklammert sind Wohngemeinschaften für unbegleitete minderjährige Flüchtlinge.
} 
Neben diesen rechtsverbindlichen internationalen und nationalen Bestimmungen existiert eine Vielzahl von „soft rules“, die von verschiedenen internationalen Gremien und Organisationen formuliert wurden:

- Der UN-Kinderrechte-Ausschuss publiziert regelmäßig Kommentare (general comments) zur KRK, die wichtige Interpretationsleitlinien enthalten. Weiters veröffentlicht er in den sog. „concluding observations“ seine Beobachtungen über den Stand der Umsetzung der Kinderrechte in den einzelnen Ländern.

- Die regelmäßige Überprüfung der Umsetzung des Europäischen Übereinkommens zur Verhütung von Folter und unmenschlicher oder erniedrigender Behandlung oder Strafe obliegt einem Komitee des Europarates, dem $\mathrm{CPT},{ }^{5}$ das über seine periodischen Staatenbesuche Berichte publiziert und Standards entwickelt. ${ }^{6}$

- Die World Medical Association hat 1998 (und neuerlich 2009) in einem Dokument (Declaration of Ottawa on Child Health 1998) [16] die Bedingungen zusammengefasst, die für eine optimale Entwicklung von Kindern notwendig sind.

- Eine internationale NGO - Defence for Children International (DCI) - arbeitet z. B. in Kooperation mit dem CPT an der konsequenten Umsetzung der Kinderrechte (vgl. Pracitcal Guide: Monitoring places where children are deprived of liberty - [10])

Diese (und weitere) Prinzipen und Regeln stellen ebenso wie bereichsspezifische Gesetze (UbG, Bundes-Kinder- und Jugendhilfegesetz) - die Grundlage der Monitoring-Tätigkeit der Kommissionen dar. Das Unterbringungsgesetz (UbG) enthält keine Altersbegrenzungen oder altersspezifischen Regelungen und gilt daher auch für Kinder- und Jugendliche. Allerdings resultieren bei Minderjährigen aus den Bestimmungen des $\mathrm{ABGB}$ zur Obsorge spezifische Bedingungen für die Anwendung des UbG, die im Einzelfall auch die Zuständigkeit des Jugendhilfeträgers einschließen können. Diese Aspekte, die nur am Rande Inhalt des NPM-Monitoring sind, verweisen auf komplexe $\mathrm{Zu}$ sammenhänge, die nicht in diesem Beitrag zu behandeln sind (s. [2]b; [8]).

Im Sinne des Präventionsauftrages wird die Beurteilungsbasis über die Rechtsnormen hinaus auch auf die Berücksichtigung von Kriterien der Betreuungsqualität erweitert. Diese Vorgangsweise stützt sich auf die Erkenntnis, dass z. B. quantitative oder qualitative Defizite im Personalbereich (oder auch im Raumangebot) sehr leicht zum Anlass einer Einschränkung von

\footnotetext{
5 European Committee for the Prevention of Torture and Inhuman or Degrading Treatment or Punishment, http://www.coe. int/en/web/cpt/home (download 28.03.17).

6 Eine Zusammenfassung dieser Standards (CPT/Inf/E (2002) 1 - Rev. 2010) ist in der Broschüre „CPT-Standards“ veröffentlicht: http://www.coe.int/en/web/cpt/standards (download 28.03.17).
}

Freiheitsrechten werden können ${ }^{7}$ Auch hier dienen sowohl gesetzliche Bestimmungen (z. B. im Krankenanstaltengesetz KAKuG) als auch verbindliche Richtlinien (z. B. Qualitätskriterien des Österreichischen Strukturplan Gesundheit) als Grundlage der Beurteilung. Wenn derartige Bestimmungen fehlen, werden auch fachspezifische Qualitätsstandards herangezogen, die beispielsweise als Konsensus-Statements von Fachgesellschaften formuliert werden.

In diesem Sinne stellt auch die wechselseitige Kooperation zwischen Kinder- und Jugendpsychiatrie einerseits und Kinder- und Jugendhilfe andererseits ein relevantes Qualitätskriterium dar, da die beiden Bereiche als kommunizierende Gefäße zu betrachten sind. Ein Defizit an Betreuungsressourcen auf der einen Seite kann ein potentielles Risiko für die Verletzung von Menschenrechten auf der anderen Seite darstellen. Verlängerungen stationärer Aufenthalte im KJP-Bereich wegen des Mangels an qualifizierten sozialtherapeutischen Wohnplätzen sind hier ebenso zu nennen wie ein mangelndes Angebot an Behandlungsplätzen in der KJP für Kinder und Jugendliche in Krisensituationen.

\section{Ausgewählte Daten aus der Kinder- und Jugend- psychiatrie}

Vorweg ist festzuhalten, dass in allen Protokollen zahlreiche positive Beobachtungen angeführt werden. Dazu zählen:

- Ein freundlicher/wertschätzender/geduldiger Umgang mit den PatientInnen, der ebenso wie die multiprofessionelle Betreuung in Kleingruppen an allen besuchten Abteilungen festgestellt wurde.

- Die Fortsetzung des Schulbesuchs im Rahmen der Heilstättenschule oder auch an der Herkunftsschule.

- An mehreren Abteilungen wurde - im Sinne von good practice-Beispielen - ein reflektiertes Krisenmanagement hervorgehoben: die Existenz von detaillierten Leitlinien zur Deeskalation und Schutzfixierung sowie eine spezielle Dokumentation zur (auch präventiven) Erfassung von Aggressionsereignissen; regelmäßige Deeskalationsschulungen; Vermeidung von oder zumindest sensibler Umgang mit Security-Einsätzen; ein klares und auf die einzelnen PatientInnen abgestimmtes Prozedere bei Isolierung/Fixierung; durchgehende 1:1 Betreuung bei Fixierungen; Veränderungen der Dienstpläne von Pflegedienst und SozialpädagogInnen mit dem Effekt einer deutlichen Reduktion von Fixierungen.

- Eine gezielte Kooperation mit Nachsorgeeinrichtungen im Kinder- und Jugendhilfebereich sowie

\footnotetext{
7 CPT/Inf/E (2002) 1 - Rev. 2010, S. 45, para 32.: „Unzulänglichkeiten können schnell zu Situationen führen, die unter den Begriff ,unmenschliche und erniedrigende Behandlung fallen.".
} 
Vernetzung mit anderen extramuralen Diensten einschließlich der Polizei.

Die zusammenfassende Inhaltsanalyse erbrachte fünf Haupt- und zwei Subkategorien, die als Rahmen für die folgende Darstellung der Evaluationsergebnisse gewählt wurden.

\section{Krisenmanagement}

- An den einzelnen Abteilungen bestehen große Unterschiede hinsichtlich des Einsatzes von SecurityDiensten. An einigen Abteilungen gibt es keine Security-Dienst, an anderen treten derartige Dienste „nicht nur punktuell bei Sicherheitsmaßnahmen in Erscheinung, sondern auch im Stationsalltag“ und sind auch bei Fixierungen anwesend, wobei die Angaben, ob sie sich dann auch aktiv beteiligen (,Hand anlegen“), nicht einheitlich sind. Auch der Umfang spezifischer Schulung der Security-Mitarbeiter ist uneinheitlich. Als Rechtsnorm gilt diesbezüglich die durchgängige Rechtsprechung des $\mathrm{OGH}$, dass alle derartigen Aktivitäten Pflegehandlungen darstellen, die dem Pflegepersonal vorbehalten sind ${ }^{8}$.

- An mehreren der besuchten Abteilungen gibt es Auszeiträume, die sich hinsichtlich Ausgestaltung und Nutzungsmodus oft weitgehend unterscheiden. Die relevantesten Differenzen betreffen die Begleitung durch StationsmitarbeiterInnen, die Überwachung (Video etc.) und die Dokumentation der Isolationsmaßnahme. In den CPT-Standards ${ }^{9}$ werden Regelungen (z. B. SOP) eingemahnt, in denen die Ziele, die Dauer, der Kontrollmodus sowie der Personenkontakt festgelegt sind. Ebenso wird eine eigenständige Dokumentation (zusätzlich zur Eintragung in der Krankengeschichte) gefordert.

\section{Freiheitsbeschränkungen, Fixierungen}

Selbstverständlich gehört die Beurteilung von Freiheitsbeschränkungen und Fixierungen zu den Kernthemen des NPM-Monitoring. Zentrale Rechtsnorm ist das Bundesverfassungsgesetz über den Schutz der persönlichen Freiheit, das in Artikel 2 Abs. 1 einen Entzug der persönlichen Freiheit u. a. aufgrund psy-

\footnotetext{
8 z. B. 7 Ob 139/14p.

9 CPT/Inf/E (2002) 1 - Rev. 2010, S. 50, para 49. „In diesem Zusammenhang soll auch auf die Absonderung (d.h. die Einzelunterbringung in einem Raum) gewalttätiger oder auf andere Weise ,nicht-steuerbarer' Patienten eingegangen werden ... Solange aber Absonderung noch angewendet wird, sollte sie Gegenstand detaillierter Grundsätze sein, die insbesondere festlegen: in welchen Fällen sie angewendet werden kann, welche Ziele durch sie angestrebt werden, die Dauer und die Notwendigkeit regelmäßiger Überprüfungen, die Existenz von angemessenem menschlichen Kontakt, die Notwendigkeit der besonderen Aufmerksamkeit des Personals. Absonderung sollte niemals als eine Bestrafung eingesetzt werden.".
}

chischer Krankheit mit Selbst- oder Fremdgefährdung (Z 5) sowie zum Zweck notwendiger Erziehungsmaßnahmen bei Minderjährigen $(\mathrm{Z} 6)$ zulässt. Artikel 1 betont in Abs. 3: „die persönliche Freiheit darf jeweils nur entzogen werden, wenn und soweit dies nicht zum Zweck der Maßnahme außer Verhältnis steht.“ und fordert dabei die Achtung der Menschenwürde und der möglichsten Schonung der Person (Abs. 4). Das UbG sowie die Standards des CPT (deren Abschnitt. III psychiatrische Einrichtungen behandelt) sind weitere wesentliche Bezugspunkte der Beurteilung. Das CPT betont die Verantwortlichkeit und Vorbildwirkung der Leitung von psychiatrischen Einrichtungen für den sorgsamen Umgang mit (unvermeidlichem) Zwang ${ }^{10}$ und fordert die 1:1-Betreuung von fixierten PatientInnen ${ }^{11}$.

Kritische Anmerkungen finden sich in den Protokollen zum Fehlen von zentralen Fixierungsregistern $^{12}$, zum Fehlen von Sitzwachen bei fixierten Patienten (einschließlich dem Fehlen von Überwachungszimmern), während die Durchführung von Nachbesprechungen von Fixierungssituationen (Personal und Patient) als good practice hervorgehoben wird. Die Sichtbarkeit nicht benützter Fixiermittel (Gurtenbetten) wird kritisch betrachtet, da sie als Mittel der Drohung und Einschüchterung wirken.

Einige Protokolle sprechen das oft schwierig zu lösende Problem der räumlichen Beschränkung (unter Anwendung des UbG) an, da KJP-Abteilungen grundsätzlich offen geführt werden. Wird die Stationstüre versperrt oder werden PatientInnen in ihrer Bewegungsfreiheit auf bestimmte Räume der Station beschränkt, so betrifft diese Maßnahme in der Regel auch andere, derzeit nicht untergebrachte, PatientInnen. Andererseits würde die Herausnahme des Patienten/der Patientin aus der Gruppe unter Verbringung in einen anderen Raum eine Isolationssituation schaffen, die in den CPT-Standards kritisch erwähnt wird. Da eine allgemeingültige, eindeutige und widerspruchsfreie Lösung dieses Problems nicht möglich erscheint, ist es notwendig, abteilungsspezifische Regeln $\mathrm{zu}$ formulieren (z. B. SOPs, die verschiedene Situationen berücksichtigen und schriftliche Begründung des Einzelfalls).

\footnotetext{
10 CPT/Inf/E (2002) 1 - Rev. 2010, S. 55, para 38: „Die Delegationen des CPT haben festgestellt, dass wenn die Krankenhausleitung eine aktive und wachsame Rolle beim Einsatz der Zwangsmaßnahmen spielt, dies meist dazu führte, dass diese Zwangsmaßnahmen seltener eingesetzt wurden.“.

11 CPT/Inf/E (2002) 1 - Rev. 2010, S. 59 para 50: „Nach Auffassung des CPT muss, wenn die Gliedmaßen eines Patienten mit Gurten oder Riemen festgeschnallt werden, ständig ein geschulter Mitarbeiter anwesend sein, um therapeutische Hilfe zu leisten. ... Die Videoüberwachung kann jedenfalls nicht die ständige Anwesenheit des Personals ersetzen.".

12 CPT/Info/E (2002) 1 - Rev.2010, S. 59, para 44 „Wenn auf Mittel körperlichen Zwanges zurückgegriffen wird, sollte dies sowohl in die Patientenakte als auch in ein geeignetes Register eingetragen werden“.
} 


\section{Aufnahme/Einlieferung}

Die verfassungsrechtliche Forderung nach Achtung der Menschenwürde wird immer wieder dann verletzt, wenn Jugendliche von der Polizei an eine KJPAbteilung gebracht werden. In den Protokollen finden sich in einigen Fällen Hinweise, dass (seitens der Polizeibeamten) die Verhältnismäßigkeit nicht gewahrt wird, wenn die Einlieferung in Handfesseln und gelegentlich auch liegend mit Bauchgurt erfolgt. Jugendliche, die aus sozialpädagogischen Wohneinrichtungen zur Aufnahme gebracht werden, kommen oft ohne sachkundige Begleitung, die erforderliche Informationen (Anamnese) liefern könnte. Dieser Umstand wird von den Kommissionen als Risiko für nachfolgende potentielle MR-Verletzungen gewertet.

\section{Aufenthaltsbedingungen in KJP (inkl. UbG-Bereich)}

Die meisten der besuchten Abteilungen befinden sich in veralteten Gebäuden, die nicht für diesen Verwendungszweck errichtet wurden und verfügen daher über keine ausreichenden Baustrukturen. Aus menschenrechtlicher Perspektive bedeutet dies, dass das Recht auf Privatsphäre ${ }^{13}$ häufig ebenso verletzt wird wie der Standard ${ }^{14}$, dass Fixierungen - sofern sie notwendig und unvermeidlich sind - in getrennten Räumen durchzuführen sind.

An manchen neu errichteten Abteilungen erfolgt die Betreuung in Kleingruppen, die auch über eine Wohnküche verfügen, in der auch gebacken und gekocht wird (good practice-Beispiel im Sinne der CPTStandards $\left.{ }^{15}\right)$.

Konflikte zwischen Peers sind naturgemäß häufige Ereignisse an KJP-Abteilungen; teilweise sind sie typisch für die Entwicklungsphase, teilweise sind sie Ausdruck der Psychopathologie. Sie stellen aber ebenso wie sexuelle Grenzüberschreitungen unter PatientInnen ein Risiko für Gewalterlebnisse dar, vor denen die Kinder und Jugendlichen geschützt werden müs-

13 WMA: Ottawa Declaration - Background Paper (2009): Dignity of the patient a. All child patients shall be treated at all times with tact and understanding and with respect for her/his dignity and privacy.

${ }^{14}$ CPT/Inf/E (2002) 1 - Rev. 2010, S. 58, para 48: 48. Im Allgemeinen sollte der Ort, an dem ein Patient fixiert wird, speziell für diesen Zweck vorgesehen sein. Er sollte sicher sein ... entsprechend beleuchtet und geheizt sein, und eine beruhigende Umgebung für den Patienten darstellen. Außerdem sollte ein fixierter Patient angemessen bekleidet und nicht den Blicken anderer Patienten ausgesetzt sein, es sei denn, er fordert dies ausdrücklich, oder bei einem Patienten ist bekannt, dass er das Zusammensein mit anderen vorzieht.

${ }^{15}$ CPT/Inf/E (2002) 1 - Rev. 2010, S. 47, para 36: „Unterbringungsstrukturen, die auf Kleingruppen basieren, sind ein entscheidender Faktor in der Erhaltung bzw. Wiederherstellung der Patientenwürde und darüber hinaus ein Schlüsselelement zur psychologischen und sozialen Wiedereingliederung der Patienten." sen. Dies wird sowohl von der $\mathrm{KRK}^{16}$ als auch vom $\mathrm{CPT}^{17}$ gefordert und mit dem Hinweis auf Prävention durch eine ausreichende Personalpräsenz verbunden. Immer wieder finden sich in den Kommissionsprotokollen (unter Bezug auf die CPT-Standards ${ }^{18}$ ) Hinweise auf ein Defizit an pädagogisch-therapeutischen Angeboten, die auf Personalmangel zurückgeführt werden.

\section{PatientInnenrechte}

Die PatientInnenrechte stehen - gerade an KJP-Abteilungen - häufig in einem Spannungsverhältnis zu den pädagogischen Aufgaben und auch zur Wahrung der schon erwähnten Rechte der MitpatientInnen. Auch wenn es oft nicht leicht ist, die entsprechenden Grenzen korrekt festzulegen, ist es notwendig, die Rechte von Kindern und Jugendlichen auch unter den Bedingungen der psychiatrischen Krankenhausbehandlung zu beachten da diese durch die UNKRK und durch nationales Verfassungsrecht geschützt sind. Dies schließt den Zugang zu Information und Partizipation ebenso ein wie das Recht auf Kommunikation nach außen und auf Beschwerdemöglichkeit. In den CPT-Standards ${ }^{19}$ wird dieser Aspekt besonders betont. Als good practice-Beispiel wird auf die einschlägige Praxis an der KJP-Klinik in Ulm hingewiesen, an der eine kindergemäße Infobroschüre mit eigenen Kapiteln über die Notaufnahme und freiheitsbeschränkende Maßnahmen publiziert wurde. ${ }^{20}$

Folgende Anmerkungen finden sich dazu in den Besuchsprotokollen:

- Handygebrauch: Einschränkungen, die es praktisch an allen Abteilungen gibt, werden vor allem mit der Gefährdung der Privatsphäre anderer PatientInnen durch das Versenden von Fotos aber auch mit der Gefahr von Mobbing (in beiden Richtungen) begründet. Die Kommissionen regen an, diesen Pro-

16 KRK Art 19: Schutz vor körperlicher oder geistiger Gewaltanwendung, Schadenszufügung oder Misshandlung, vor Verwahrlosung oder Vernachlässigung, vor schlechter Behandlung oder Ausbeutung einschließlich des sexuellen Missbrauchs.

17 CPT S. 45, 30. Es ist ebenfalls unabdingbar, dass angemessene Vorkehrungen bestehen, um bestimmte Psychiatriepatienten vor anderen Patienten zu schützen, die sie verletzen könnten. Dies erfordert unter anderem eine angemessene Personalpräsenz zu jeder Zeit, auch nachts und am Wochenende. Darüber hinaus sollten spezielle Vorkehrungen für besonders verletzliche Patienten getroffen werden.

18 CPT/Inf/E (2002) 1 - Rev. 2010, S. 47, para 37: „Es sollte ein großes Angebot rehabilitativer und therapeutischer Aktivitäten geben."

${ }^{19}$ CPT/Inf/E (2002) 1-Rev. 2010, S. 52, para 53: „Eine einführende Broschüre über die Abläufe der Einrichtung und die Rechte der Patienten sollte sowohl jedem Patienten bei seiner Ankunft als auch seiner Familie übergeben werden. Alle Patienten, die die Broschüre nicht verstehen können, sollten geeigneten Beistand erhalten."

${ }^{20}$ http://www.uniklinik-ulm.de/fileadmin/Zentrale_Seiten/ Pressestelle/Presse_2010/Was_ist_denn_schon_normal.pdf (download 28.03.17). 
blemen durch medienpädagogische Konzepte $\mathrm{zu}$ begegnen.

- Kontakt nach außen: Hinweis auf UbG $\$ 34 / 1$. Die Möglichkeit, täglich zu telefonieren, wird positiv hervorgehoben. Ebenso die allgemein großzügigen Besuchsregelungen, die nur im UbG-Bereich bzw. nur nach Anordnung des Jugendamtes Einschränkungen erfahren. Auch Wochenendausgänge zählen zu den positiven Beobachtungen.

- Beschwerdemöglichkeiten: Diesem Recht wird oft nicht ausreichend Beachtung geschenkt. Jedenfalls reicht die Beschwerdemöglichkeit der Eltern - zumindest bei > 14-Jährigen - nicht aus. Auch Kindern, deren Vertrauensverhältnis $\mathrm{zu}$ den Eltern beeinträchtigt ist, muss ein anderer Weg der Beschwerde offenstehen. Das Wissen über formalisierte Beschwerdeoptionen ist Teil des Rechts auf Information.

- Der (z. B. telefonische) Zugang zur regionalen Kinder- und Jugendanwaltschaft und zur Patientenanwaltschaft ist durch Aushang im Lebensbereich der PatientInnen zu gewährleisten.

- Das Recht auf Partizipation sollte durch ein Stationsparlament, Patientenforum oder ähnliche Strukturen gewährleistet sein.

\section{Strukturelle Versorgungsdefizite}

Ein nahezu durchgängiges Thema in den Protokollen ist die Kritik an den defizitären Rahmenbedingungen der KJP-Versorgung einschließlich des Hinweises, dass diese Defizite großen Einfluss auf die Betreuungsbedingungen an den Abteilungen und damit auf die Menschenrechtskonformität haben. Die Tatsache, dass die Kinder- und Jugendpsychiatrie in Österreich erst 2007 als eigenständiges Sonderfach etabliert wurde, kann die Versorgungsdefizite zwar teilweise erklären, aber nicht entschuldigen. Die Planungsgrundlagen der KJP-Versorgung in Österreich wurden in der Vorbereitung des Österreichischen Strukturplan Gesundheit (ÖSG) seit dem Jahre 2005 entwickelt und sind in der Version 2012 (unverändert) dargestellt. Weitere 5 Jahre später sind alle Bundesländer von diesen Strukturkriterien noch immer weit entfernt. In den Kommissionsprotokollen werden - auf der Grundlage der Informationen aus den besuchten Abteilungen - Defizite auf allen Ebenen beschrieben: Defizite an ambulanten, teilstationären und stationären Behandlungsplätzen, ein Mangel an FachärztInnen im Allgemeinen und an niedergelassenen FachärztInnen im Besonderen. Diese Defizite beeinflussen die vor- und nachstationäre Behandlung, die Aufenthaltsdauer ${ }^{21}$, die Qualität der stationären Betreuung und das Nahtstellenmanagement (zur Erwachsenenpsychiatrie, zur Kinder- und Jugendhilfe). Diese Defizite stehen im Widerspruch zu den relevanten Kinderrechts- und Gesundheitsstandards (KRK ${ }^{22}$, UN-Kinderrechteausschuss General Comment Nr. 15²3, WMA-Declaration of Ottawa ${ }^{24}$, ÖSG ${ }^{25}$ ).

Beispielhafte Kritikpunkte aus den Protokollen:

- Verlängerung der stationären Aufenthalte mit dysfunktionaler Bindung an die Klinik und lange Wartezeiten auf stationäre Neuaufnahmen mit Tendenz zur Chronifizierung von Störungsbildern

- Fehlen extramuraler Nachbetreuung im räumlichen Nahebereich zum Wohnort

- Mangel an kinderpsychiatrischen Nacht- und Wochenenddiensten

- Defizite an fachspezifischer Kompetenz bei UbGAufnahmeuntersuchungen ${ }^{26}$

- Stationssperren aus Kapazitätsgründen während der Urlaubszeit

\section{(Nicht-)Einhaltung des Trennungsgebots}

Der Trennung der Kinder und Jugendlichen von Erwachsenen im stationären Bereich wird in allen Beurteilungsrichtlinien große Bedeutung zugemessen

21 CPT/Inf/E (2002) 1 - Rev. 2010, S. 53, para 57. „Auch wenn die unfreiwillige Unterbringung eines Patienten nicht mehr erforderlich ist, kann er gleichwohl einer Behandlung und/oder einer geschützten Umgebung außerhalb der Einrichtung bedürfen. In diesem Zusammenhang hat das CPT in einer Anzahl von Ländern festgestellt, dass Patienten, deren Geisteszustand es nicht länger erforderte, in einer psychiatrischen Einrichtung festgehalten zu werden, gleichwohl in solchen Einrichtungen verblieben, weil es außerhalb keine angemessene Betreuung/Unterbringung gab. Wenn Personen in Freiheitsentzug bleiben müssen, weil geeignete externe Einrichtungen fehlen, so ist dies eine höchst bedenkliche Sachlage.“

22 Vgl. KRKArt. 24: Recht des Kindes auf das erreichbare Höchstmaß an Gesundheit sowie auf Inanspruchnahme von Einrichtungen zur Behandlung von Krankheiten und zur Wiederherstellung der Gesundheit. Bemühung sicherzustellen, dass keinem Kind das Recht auf Zugang zu derartigen Gesundheitsdiensten vorenthalten wird.

$23 \mathrm{CRC} / \mathrm{C} / \mathrm{GC} / 15$ para 104. „In their decisions about budget allocation and spending, States should strive to ensure availability, accessibility, acceptability and quality of essential children's health services for all, without discrimination."

24 Health resources available to all a. Every child shall be provided with adequate medical assistance and health care, with emphasis on primary/basic health care, pertinent psychiatric care for those requiring it, $b$. Care appropriate to the needs of specially-abled children shall be available. h. Child and adolescent health services will be adequately funded and provided.

25 ÖSG 2012, S. 55 ff. 2.4.2.3 Kinder- und Jugendpsychiatrie (KJP).

26 Protokoll (08/2016): Wenn der diensthabende Facharzt eine ambulante Betreuung als ausreichend empfinde, gebe es keine weiterführende Begutachtung durch einen Kinderpsychiater. Dies könne im Einzelfall auch rechtliche Probleme verursachen. 
$\left(\mathrm{KRK}^{27}, \mathrm{WMA}^{28}\right.$, Empfehlungen der Volksanwaltschaft ${ }^{29}$, $\left.\mathrm{OGH}^{30}\right)$. Nichtsdestoweniger kommt es in ganz Österreich immer wieder zu stationären Aufnahmen von Kindern und Jugendlichen (mit und ohne UbG) auf erwachsenenpsychiatrischen Stationen. Die Transferierungen aus der KJP-Uni-Klinik Wien auf erwachsenenpsychiatrische Abteilungen haben in den Jahren 2014/15 wesentlich zugenommen (3-fach).

Protokollbeispiele:

- Wien (02/2016): Wenn aus Kapazitätsgründen vorübergehend auf Ressourcen der Erwachsenenpsychiatrie zurückgegriffen werden muss, sollte dies als absolute Notlösung verstanden werden und eine Rücktransferierung der jugendlichen PatientInnen im kürzest möglichen Zeitraum erfolgen. Eine Verweildauer von 14 Tagen (wie im Fall von Fr. $\mathrm{xxx}$ ) erscheint jedenfalls unverhältnismäßig und sollte vermieden werden.

- Innsbruck (07/2016): 11-Jähriger mit Security in den UbG-Bereich an der geschlossenen Männerabteilung begleitet

- Eine altersspezifische Betreuung auf erwachsenenpsychiatrischen Abteilungen findet - wenn überhaupt - nur im Rahmen eines konsiliarärztlichen Kooperationmodells statt (z. B. Wien, Tulln, Innsbruck) - die sonstigen altersspezifischen Angebote (pädagogische Gruppen, Heilstättenschule etc.) stehen nicht zur Verfügung.

- Gibt es ausreichend Bewusstsein und Schutzvorkehrungen insbesondere zum Schutz von Opfern sexueller Gewalt? (Wien 05/2015)

Für die stationäre Betreuung jugendlicher PatientInnen aus dem Bereich der forensischen Psychiatrie gibt es keine einheitlichen Regelungen. An einigen KJPAbteilungen (z. B. Christian Doppler-Klinik, Salzburg) werden diese PatientInnen grundsätzlich mitbetreut; meist sind diese Abteilungen aber zu klein und nicht hinreichend ausgestattet, sodass auch hier Verlegungen in den erwachsenenpsychiatrischen Bereich erfolgen.

\footnotetext{
27 KRK Art. 37 „Die Vertragsstaaten stellen sicher, dass jedes Kind, dem die Freiheit entzogen ist, menschlich und mit Achtung vor der dem Menschen innewohnenden Würde und unter Berücksichtigung der Bedürfnisse von Personen seines Alters behandelt wird. Insbesondere ist jedes Kind, dem die Freiheit entzogen ist, von Erwachsenen zu trennen, sofern nicht ein anderes Vorgehen als dem Wohl des Kindes dienlich erachtet wird."

28 WMA-Declaration of Ottawa: Hospitalization a. Whenever possible a child admitted to hospital should be accommodated in an environment designed and equipped to suit her/his age and health status.

29 VA-Empfehlungen: Kinder und Jugendliche dürfen nicht in der Erwachsenenpsychiatrie behandelt und untergebracht werden; das stellt auch nach Ansicht des CPT eine Verletzung präventiver menschenrechtlicher und fachlicher Standards dar.

$307 \mathrm{Ob}$ 107/14g zu Trennungsgebot (betr. forensisch-psychiatrischen Bereich).
}

\section{Nahtstellen zur Kinder- und Jugendhilfe}

Monitoringbesuche an KJP-Abteilungen erfassen auch die Nahtstelle zu Einrichtungen der Kinder- und Jugendhilfe, da viele KJP-PatientInnen einer poststationären Betreuung (Sozialarbeit, Sozialpädagogik) bedürfen. Häufig genannte Probleme sind lange Wartezeiten auf sozialtherapeutische Betreuungsangebote (Wohnplätze, intensive Familienbetreuung) mit hoher Betreuungsintensität oder auch der Verlust eines WG-Platzes im Zuge einer stationären KJP-Aufnahme. Helferkonferenzen können geeignete Instrumente des Nahtstellenmanagements sein um derartige Probleme $\mathrm{zu}$ reduzieren.

\section{Daten aus der Kinder- und Jugendhilfe}

Die Darstellung der Ergebnisse erfolgt im Überblick, da die Heterogenität der Einrichtungstypen keine sinnvolle Kategorienbildung zulässt.

Die öffentliche Diskussion über Gewalt in Heimen, die ab 2010 in Österreich stattgefunden hat [4], belegt die Bedeutung eines externen Monitoringprozesses in diesem Bereich. Sowohl die UN-KRK als auch das BVG-Kinderrechte enthalten grundlegende Bestimmungen, die essentielle Maßstäbe des Monitorings sind. Darüber hinaus kommt im breit gefächerten Spektrum der Monitoring-Themen der Berücksichtigung von Qualitätsstandards als Präventionsinstrument besondere Bedeutung $\mathrm{zu}$.

Folgende Monitoring-Themen sind unmittelbar aus UN-Kinderrechtekonvention und dem BVG Kinderrechte ableitbar:

- Anspruch auf Schutz, Fürsorge und Wohlergehen (BVG-KR Art. 1)

- Schutz vor Gewalt und erniedrigender Behandlung (UN-KRK Art. 19(1,2), 20, 37; BVG-KR Art 5(1), 6),

- Kontaktmöglichkeiten nach außen (KRK Art. 9, Abs 3; BVG KR Art. 2(1)),

- Freizeitaktivitäten (UN-KRK Art. 31(1,2)),

- Privatsphäre (UN-KRKArt. 16(1,2)),

- Religion (UN-KRK Art. $14(1,2,3))$,

- Bildung (UN-KRK Art. 28(1)),

- Partizipation und Meinungsäußerung (UN-KRK Art. 12(1), 13(1); BVG-KR Art. 4),

- Recht auf Gesundheit (UN-KRK Art. 24)

- Wiedereingliederung nach Traumatisierung (UNKRK Art. 39)

Die Beurteilung der Betreuungsqualität erfolgt vor allem anhand der Quality4Children-Standards, die von Fachorganisationen aus 32 europäischen Ländern mit dem Ziel, die Situation und Entwicklungschancen von fremd untergebrachten Kindern zu verbessern, 
gemeinsam formuliert wurden. ${ }^{31}$ Auch die Empfehlungen der Volksanwaltschaft ${ }^{32}$ stellen Bezugspunkte für die Beurteilung dar.

Vor dem Hintergrund der Erkenntnisse über die schwerwiegenden Defizite der Heimerziehung in den 1950 -er bis 1980-er Jahren [7, 15] kommt einigen Themen besondere Bedeutung zu. Dazu gehören die Möglichkeiten des Kontakts nach außen im Allgemeinen und $\mathrm{zu}$ externen Ombudsstellen im Besonderen sowie der Schutz vor (sexueller) Gewalt.

Protokollbeispiele:

- Die Möglichkeiten des selbständigen Verlassens der Einrichtung streuen von „offenes Haus, abends Türe versperrt“ über „geregelte Ausgangszeiten (altersunabhängig 1,5 h tgl.)“ bis zu „restriktive Ausgangszeiten der Hausordnung stehen im Widerspruch zum Jugendschutzgesetz des Landes“ und „BewohnerInnen haben keinen Schlüssel, die Fenster, aus denen Ausstieg möglich ist, sind vergittert. In Summe haftähnliche Anhaltung." Selbstverständlich steht dieser Aspekt in einem Spannungsverhältnis zur Aufsichtspflicht, die altersabhängig (unter Berücksichtigung individueller Kompetenzen) zu gestalten ist. Die Kommissionen achten auf die Existenz transparenter und reflektierter Regeln.

- Die Möglichkeiten, zu externen Ombuds- und Beratungsstellen Kontakt aufzunehmen, werden von den Kommissionen besonders aufmerksam beachtet. In diesem Bereich hat die Tätigkeit des NPM einen deutlichen Beitrag zur Bewusstseinsbildung über die Notwendigkeit von Beschwerdemöglichkeiten geleistet. In manchen Einrichtungen finden Sprechtage der regionalen Kinder- und Jugendanwaltschaften statt, in anderen sind zumindest deren Erreichbarkeitsdaten sichtbar angeschlagen. Bedauerlicherweise finden sich aber auch gegenteilige Protokolleinträge: „Keinerlei Informationen oder Aushänge im Haus. Keine Aufklärung über Rechte oder Rechtsvertretung an die Klientinnen und Vertretungsmöglichkeiten sind auch unbekannt.“

- Sowohl die Besuchsregelungen (Eltern, Vertrauenspersonen, FreundInnen) als auch die HandyNutzungsregeln zeigen im Einzelnen große Unterschiede. Restriktive Regeln sind mittlerweile allerdings die Ausnahme. Die Kommissionen betonen immer wieder die Notwendigkeit medienpädagogischer Konzepte anstatt restriktiver Nutzungsregeln.

- Gewaltprävention war in den Jahren 2013/14 ein Prüfschwerpunkt der Volksanwaltschaft, 2015 lag

\footnotetext{
31 https://www.fice.at/quality-for-children. Download der Standards unter: http://www.sos-childrensvillages.org/getmedia/ 79e5ea1b-d0b1-43df-8e13-5e37b7e45fa7/Quality_Deutsch.pdf (download 31.03.17).

32 https://volksanwaltschaft.gv.at/downloads/34ck2/empfeh lungsliste_201608_update.pdf (download 25.03.2017).
}

der Fokus auf der Prävention von sexueller Gewalt. ${ }^{33}$ Auch hier ist $\mathrm{zu}$ beobachten, dass diese Schwerpunktsetzung des NPM einen Beitrag zu wachsender Aufmerksamkeit in den Einrichtungen leisten konnte. Dennoch beschreiben die Besuchsprotokolle noch immer ein breites Spektrum: „Reflektierter Umgang mit Gewaltvorkommnissen“ und „Fortbildungen im Bereich Gewaltprävention“ werden positiv hervorgehoben; in anderen WG's wird kritisch festgehalten: „Fehlen von Deeskalationsfortbildungen und schriftlichen Konzepten zur Prävention von (sexueller) Gewalt“, „Kein gemeinsames Verständnis, ab wann Gewalt beginnt“. Mobbing und Bullying werden bedauerlicherweise nur selten als pädagogische Probleme explizit thematisiert. Der pädagogische Umgang mit Krisensituationen stellt unter Umständen auch die Option der passageren Freiheitsbeschränkung (Festhalten, Einsperren) und die Kooperation mit der Polizei zur Diskussion. Da es für diese Situationen keine einfachen Regeln geben kann, soll ein Protokollbeispiel als Orientierungshilfe zitiert werden: „Bei aggressivem Verhalten wird ein Protokoll mit genauer Analyse des Verhaltens und der näheren Umstände angelegt um danach die Bearbeitung des Verhaltens in den Betreuungsplan aufzunehmen. Alle Probleme werden zeitnah und transparent besprochen und behandelt“. Als Instrument zur Strukturierung des Umgangs mit grenzverletzendem Verhalten und Gewaltphänomenen wird seitens des NPM auf den „Bündner Standard“34 hingewiesen. Die Feststellung „Festhalten/Fixierung wurde von Praktikant durchgeführt" ist jedenfalls eine negative Konnotation. Eine strukturierte Kooperation mit der regionalen Polizeidienststelle wird als sinnvolle Strategie gewertet.

- Die Formulierung des Rechtes auf Partizipation im BVG-Kinderrechte ${ }^{35}$ ist als besonders bedeutsam einzuschätzen. Die Umsetzung dieses Rechts wird in den Protokollen positiv betont: „Regelmäßiges Kinderforum“, „Leitfaden zum Thema Beteiligung, verschiedene Informations- und Partizipationsebenen in der Einrichtung vorhanden“.
33 Vgl. dazu Parlamentsbericht 2014, Band 2 NPM, S. $58 \mathrm{ff}$ und Parlamentsbericht 2015 Präventive Menschenrechtskonrolle, S. $68 \mathrm{ff}$. https://volksanwaltschaft.gv.at/downloads/b5cgi/ Parlamentsbericht\%202014\%20Band\%20II.pdf (download 31.03. 2017).

34 http://www.buendner-standard.ch/home/ (download 31.03. 2017).

35 Artikel 4: Jedes Kind hat das Recht auf angemessene Beteiligung und Berücksichtigung seiner Meinung in allen das Kind betreffenden Angelegenheiten, in einer seinem Alter und seiner Entwicklung entsprechenden Weise. 
- Obwohl das Recht des Kindes auf Schutz ihrer Privatsphäre bereits seit 1989 in der UN-KRK ${ }^{36}$ verankert ist, ist der Prozess der Bewusstseinsbildung im pädagogischen Bereich noch unzureichend entwickelt. In vielen Einrichtungen sind die Zimmer ebensowenig von innen verschließbar wie Duschund Baderäume, verschließbare Kästchen fehlen, Zimmerkontrollen erfolgen häufig unangemeldet und ohne Wissen der BewohnerInnen.

- In nahezu allen Protokollen findet sich eine positive Beurteilung der Umsetzung des Rechts auf Bildung. In vielen Einrichtungen treffen die Kommissionen auf intensive Bemühungen und teilweise großen Personalaufwand, um den Schulbesuch sicherzustellen bzw. bei längerfristiger Schulverweigerung alternative Bildungsmöglichkeiten anzubieten.

- Die Einlösung des Rechts auf Gesundheit stößt dort an Grenzen, wo die Angebote an Psychotherapie und die ausreichende Kooperation mit KJP-Einrichtungen fehlen. Hier handelt es sich meist um strukturelle Probleme, die oft außerhalb des Einflussbereichs der KJH-Einrichtungen liegen. Das Management von erforderlichen Medikamenten, deren Aufbewahrung und Verabreichung, liegt hingegen im unmittelbaren Verantwortungsbereich der Einrichtung. Aus vielen Protokollen ist ersichtlich, dass sowohl die Aufbewahrung (keine versperrten Schränke), als auch die Vorbereitung der Ausgabe (kein adäquates Blistersystem) sowie die Dokumentation der Anordnung und Ausgabe von Psychopharmaka große Defizite aufweisen. Im Falle der ärztlich angeordneten Bedarfsmedikation ist es unumgänglich, dass diese Anordnung mit einer klaren und praktisch nutzbaren Beschreibung der Bedarfssituation verbunden ist und auch die Verabreichungssituation ausreichend dokumentiert wird. Die PädagogInnen, die mit dieser Aufgabe betraut werden, müssen über eine fachliche Zusatzqualifikation ${ }^{37}$ verfügen.

Die Beurteilung der Qualität der Betreuungsstandards wird - wie schon früher dargestellt - als bedeutsames Präventionsinstrument gewertet, das auch fernab unmittelbarer Verletzung oder Gefährdung von Menschen- und Kinderrechten zur Anwendung gelangt. Die Quality4Children-Standards, bieten dafür einen wichtigen Raster. Folgende Beispiele fallen in diesen Bereich:

Die Erstellung individueller Betreuungspläne ${ }^{38}$ sowie die schriftliche und damit nachvollziehbare Dokumentation des Betreuungsprozesses sind Teil der

\footnotetext{
36 Artikel 16. 1. Kein Kind darf willkürlichen oder rechtswidrigen Eingriffen in sein Privatleben, seine Familie, seine Wohnung oder seinen Schriftverkehr oder rechtswidrigen Beeinträchtigungen seiner Ehre und seines Rufes ausgesetzt werden.

37 Z. B. Ausbildungsmodul „Unterstützung bei der Basisversorgung“.

38 Q4C Standard 6: Der Betreuungsprozess während der Fremdunterbringung folgt einem individuellen Betreuungsplan.
}

NPM-Beurteilung. Ein negatives Protokollbeispiel lautet: „Keine laufende personen- oder prozessbezogene Dokumentation hinsichtlich Förderungs- und Betreuungsplanung. Keine Zielvereinbarungen, keine Nachvollziehbarkeit von Planung und Umsetzung individueller Entwicklungsprozessen. Keine individuelle Planung oder Mitgestaltungsmöglichkeit.“

Weitere Bereiche, die ebenfalls zum Beurteilungsspektrum des NPM gehören, weisen in den Protokollen so große Streuungen auf, dass eine Zusammenfassung nicht sinnvoll ist; sie sollen an dieser Stelle nur erwähnt werden: Die Personalqualität (Ausbildungsniveau, Fortbildungsmöglichkeiten) und die Arbeitsbedingungen (Dienstpläne, Supervisionsangebote), die Gestaltung der allgemeinen Lebensbedingungen (Hausregeln, pädagogische Sanktionen, Sexualerziehung etc.) sowie die hygienischen Bedingungen, die Lage und Erreichbarkeit der Einrichtungen, die Arbeit mit den Herkunftsfamilien, der Umgang mit religiösen Ritualen (z. B. bei MigrantInnen und unbegleiteten minderjährigen Flüchtlingen).

\section{Diskussion}

Das menschenrechtliche Monitoring, das durch das OPCAT-Gesetz in Österreich etabliert wurde, stellt eine spezifische Form des Grundrechtsschutzes dar. Klaushofer [12] stellt dem „klassischen individuellen Grundrechtsschutz“ den „objektiven Grundrechtsschutz“ gegenüber, der zwar keinen Individualrechtsschutz bieten kann, aber größere Breitenwirkung hat: „Es findet eine institutionelle Auseinandersetzung mit anderen Staatsorganen und privaten Einrichtungen statt und nicht bloß eine parteien- und verfahrensmäßige Abwicklung“ (S. 113). In einem Beispiel aus dem Kinder- und Jugendhilfebereich weist er darauf hin, dass Betroffene oft weder das Wissen noch die faktische Möglichkeit einer Beschwerde haben und menschenrechtlich Probleme daher oft unentdeckt bleiben und resümiert: „Grundrechtsschutz durch Präventionsmechanismen kann individuelle Rechtsansprüche nie ersetzen, aber oft viel schneller und breitenwirksamer zu einer Verbesserung der Menschenrechtssituation beitragen als dieser es je vermag." (S. 113).

Ein Vergleich der referierten Ergebnisse mit internationalen Entwicklungen ist nur punktuell, ein direkter Datenvergleich aufgrund fehlender einschlägiger Publikationen nicht möglich.

Das Optional Protocol (OPCAT) wurde bisher von 83 Staaten ratifiziert, die damit die Verpflichtung zur Einrichtung eines Nationalen Präventionsmechanismus (mit unterschiedlichen konkreten Ausformungen) eingegangen sind. Der österreichische NPM hat aufgrund seiner verfassungsrechtlichen Konstruktion insbesondere im Kinder- und Jugendbereich (aber auch im Bereich der Behindertenbetreuung) im internationalen Kontext eine Pionierrolle übernommen, da in anderen Ländern Besuche in diesem Bereich 
nicht oder in deutlich geringerem Umfang stattfinden. Im Vergleich zu Deutschland und der Schweiz ist die Besuchstätigkeit der Volksanwaltschafts-Kommissionen in Österreich im Kinder- und Jugendbereich wesentlich umfangreicher.

In der Schweiz wurde die „Nationale Kommission zur Verhütung von Folter (NKVF)“39 eingerichtet, die aus 9 Mitgliedern besteht. Die Kommission veröffentlicht Berichte und Stellungnahmen zu Besuchen in Justizeinrichtungen.

Deutschland hat eine „Nationale Stelle zur Verhütung von Folter" ${ }^{\prime 40}$ eingerichtet, die aus einer Bundesstelle und einer Länderkommission besteht. Im Jahresbericht 2015 [14] des deutschen NPM wird erstmals über Besuche in psychiatrischen Kliniken und Einrichtungen der Kinder- und Jugendhilfe berichtet, da die Besuchstätigkeit in den Vorjahren (ab 2009) auf Einrichtungen der Polizei und Justiz beschränkt waren. Kritisch erwähnt wird dort der Mangel an Räumlichkeiten für pädagogisch-therapeutische $\mathrm{Ar}$ beit. Positiv hervorgehoben werden der empathische Umgang der Mitarbeiterinnen mit den Jugendlichen sowie das Fehlen von Time-out-Räumen. Besuche in kinder- und jugendpsychiatrische Kliniken werden nicht angeführt.

Aus den referierten Evaluationsergebnissen sind verschiedene Empfehlungen abzuleiten:

Ein Ausgleich der Versorgungsdefizite im kinderund jugendpsychiatrischen Bereich erfordert Entscheidungen auf fachpolitischer und gesellschaftspolitischer Ebene. Seit 2007, dem Jahr der Etablierung des Sonderfaches Kinder- und Jugendpsychiatrie, sind hier zweifellos Entwicklungen zu beobachten, die aber keineswegs ausreichen, um diese Defizite im stationären und ambulanten Bereich zu kompensieren.

An MitarbeiterInnen von KJP-Krankenhausabteilungen ist die Empfehlung zu richten, im Sinne der verfassungsrechtlich festgeschriebenen Kinderrechte die Patientenrechte auch in Alltagssituationen zu beachten und eine spezifische Sensibilität für das Krisenmanagement und in der Anwendung freiheitsbeschränkender Maßnahmen zu fördern.

In Wohneinrichtungen der Kinder- und Jugendhilfe ist den Aspekten der Gewaltprävention, der Schaffung adäquater Beschwerdemöglichkeiten (als eigenständiges Kinderrecht) und der Partizipation besonderes Augenmerk zu schenken. Auch dies ist als Empfehlung an die dort tätigen MitarbeiterInnen zu werten.

Acknowledgements Open access funding provided by Medical University of Vienna.

Interessenkonflikt E. Berger und C. Paar geben an, dass kein Interessenkonflikt besteht.

39 https://www.nkvf.admin.ch/nkvf/de/home.html (download 31.03.2017).

${ }^{40}$ http://www.nationale-stelle.de/home.html (download 03.04. 17).
Open Access Dieser Artikel wird unter der Creative Commons Namensnennung 4.0 International Lizenz (http:// creativecommons.org/licenses/by/4.0/deed.de) veröffentlicht, welche die Nutzung, Vervielfältigung, Bearbeitung, Verbreitung und Wiedergabe in jeglichem Medium und Format erlaubt, sofern Sie den/die ursprünglichen Autor(en) und die Quelle ordnungsgemäß nennen, einen Link zur Creative Commons Lizenz beifügen und angeben, ob Änderungen vorgenommen wurden.

\section{Literatur}

1. Berger E. Jugendwohlfahrt und Fürsorge im Nationalsozialismus. In: Bundesjugendvertretung (Hrsg.). Geraubte Kindheit. Kinder und Jugendliche im Nationalsozialismus. Wien: Edition Mauthausen; 2010a. S. 87-96.

2. Berger E. Spezifische Probleme der Unterbringung Minderjähriger. Interdiszipl Zeitschr $\mathrm{F}$ Familienrecht. 2010b;5:18-20. Spezial Juli 2010.

3. BergerE.DieInnsbruckerKinderpsychiatrie/Heilpädagogik im Kontext der Entwicklung des Faches Kinderpsychiatrie. In: Medizin-Historische ExpertInnenkommission (Hrsg.). Die Innsbrucker Kinderbeobachtungsstation von Maria Nowak-Vogl. Innsbruck: Med. Univ. Innsbruck; 2013. S. 50-6.

4. Berger E. Gewalt in pädagogischen Institutionen in Österreich.In:FegertJM, WolfM(Hrsg.).Kompendium „,Sexueller Missbrauch in Institutionen“. Weinheim u. Basel: Beltz Juventa; 2015. S. 697-706.

5. Berger E. Die Kinderpsychiatrie in Österreich 1945-1975. Entwicklungen zwischen historischer Hypothek und sozialpsychiatrischem Anspruch. In: Gabriel E, Dietrich-Daum E, Lobenwein E, Watzka C (Hrsg.). Virus. Beiträge zur Sozialgeschichte der Medizin. 14. Aufl. 2016. S. 239-48.

6. Berger E, Aichhorn W, Friedrich MH, Fiala-Preinsperger S, Leixnering W, Mangold B, Spiel G, Thun-Hohenstein L. Kinder- und jugendpsychiatrische Versorgung in Österreich. Neuropsychiatrie. 2006;20:86-90.

7. Berger E, Katschnig T. Gewalt in Wiener Heimen zwischen 1945 und 1990 - eine retrospektive Studie aus psychotraumatologischer Perspektive. Neuropsychiatrie. 2013;27(4):188-95.

8. Berger E, Spiel G. Freiheitsentziehende Maßnahmen. In: Kerbl R, Thun-Hohenstein L, Damm L, Waldhauser F (Hrsg.). „Kind und Recht“ (3.Jahrestagung Politische Kindermedizin). Wien NewYork: Springer;2010. S. 93-102.

9. Rechtsinformationssystem des Bundes (RIS). Bundesverfassungsgesetz über die Rechte von Kindern. 2011. https:/ / www.ris.bka.gv.at/GeltendeFassung.wxe?Abfrage=Bundes normen\&amp;Gesetzesnummer=20007136. Zugegriffen: 14. Jan. 2017.

10. Defence for Children International (DCI). Pracitcal Guide: Monitoring places where children are deprived of liberty. 2016. www.defenceforchildren.org/monitoring-placeswhere-children-are-deprived-of-liberty-a-practicalguide.Zugegriffen: 14. Jan. 2017.

11. GoffmanE.Asyle-überdiesozialeSituation psychiatrischer Patientenund anderer Insasse. Frankfurt/Main:Suhrkamp; 1973. Orig. Amerikanisch 1961.

12. Klaushofer R. Grundrechtsschutz durch die Volksanwaltschaft. Ein Bericht aus der Praxis des objektiven Grundrechtsschutzes. In: Pabel K, Raschauer N (Hrsg.). Die Organisation des Grundrechtsschutzes. Nationale und europäischePerspektiven. Wien:VerlagÖsterreich;2014.

13. Mayring P. Qualitative Inhaltsanalyse. 12. Aufl. Weinheim: Beltz;2015. 
14. Nationale Stelle zur Verhütung von Folter. Jahresbericht der Bundesstelle und der Länderkommission. 2015. http://www.nationale-stelle.de/fileadmin/dateiablage/ Dokumente/Berichte/Jahresberichte/Jahresbericht_2015_ Nationale_Stelle.pdf.Zugegriffen:25. März2017.
15. Sieder R, Smioski A. Der Kindheit beraubt. Gewalt in den Erziehungsheimen der Stadt Wien. Innsbruck: StudienVerlag. 2012

16. WMA. Declaration of Ottawa on child health. 1998. http:// www.wma.net/en/30publications/10policies/c4/. Zugegriffen: 14.Jan. 2017. 